\title{
Cancer-specific Therapeutic Potential of Resveratrol: Metabolic Approach against Hallmarks of Cancer
}

\author{
Dong Hoon Suh", Mi-Kyung Kim², Hee Seung Kim², Hyun Hoon Chung², Yong Sang \\ Song $^{2,3,4, *}$
}

${ }^{1}$ Department of Obstetrics and Gynecology, Seoul National University Bundang Hospital, Seongnam, Korea; ${ }^{2}$ Department of Obstetrics and Gynecology, Seoul National University College of Medicine, Seoul, Korea; ${ }^{3}$ Cancer Research Institute, Seoul National University College of Medicine, Seoul, Korea; ${ }^{4}$ Major in Biomodulation, World Class University, Seoul National University, Seoul, Korea

Corresponding Author: Yong Sang Song, Department of Obstetrics and Gynecology, Seoul National University College of Medicine, 101 Daehak-ro Jongno-gu, Seoul 110-744, Korea

Submission date: May 2, 2013; Acceptance date: August 21, 2013; Publication date: August 26, 2013

\begin{abstract}
:
Cancer hallmarks include evading apoptosis, limitless replicative potential, sustained angiogenesis, tissue invasion and metastasis. Cancer cells undergo metabolic reprogramming and inevitably take advantage of glycolysis to meet the increased metabolic demand: rapid energy generation and macromolecular synthesis. Resveratrol, a polyphenolic phytoalexin, is known to exhibit pleiotropic anti-cancer effects most of which are linked to metabolic reprogramming in cancer cells. This review summarizes various anti-cancer effects of resveratrol in the context of cancer hallmarks in relation to metabolic reprogramming.
\end{abstract}

\section{INTRODUCTION:}

Tumor cells rely on glycolysis rather than oxidative phosphorylation for ATP production even in the presence of oxygen, this is called the Warburg effect. Since the proposal of metabolic shift from aerobic respiration to aerobic glycolysis in neoplastic cells [1], supporting evidence has been accumulated in many types of cancers [2]. The Warburg effect in the context of concerted changes in energy metabolism including mitochondrial function for carcinogenesis is often referred to as metabolic reprogramming [3]. Although most of the exact molecular mechanisms underlying metabolic reprogramming are needed to be elucidated, many anti-cancer agents targeting the metabolic reprogramming are under investigation in the preclinical and clinical setting [4]. 
Among the agents is a naturally occurring compound, phytochemicals, which comprise different classes such as terpenoids, glucosinolates, anthraquinones, and polyphenolics [5]. Resveratrol (3, 5, 4'-trihydroxy-trans-stilbene), a biologically potent member of polyphenolics, was known as one of phytoalexins [6]. Tremendous interest in resveratrol of earlier days was mainly due to the epidemiological finding of so-called "French paradox", i.e., despite high fat intake, mortality from coronary heart disease of people who regularly drink wine is low [7]. Parallel to the anti-cardiovascular effect, anti-cancer effect of resveratrol has been extensively studied for the past several years. An enormous amount of evidence has proposed many different mechanisms associated with resveratrol, including anti-oxidant and anti-mutagenic properties [8]. Despite the fact that a lot of anti-cancer mechanisms of resveratrol are still largely obscure, the pleiotropic nature of its anti-cancer effect is noteworthy (figure 1). This review, herein, summarizes various anti-cancer effects of resveratrol in the context of cancer hallmarks in relation to metabolic reprogramming.

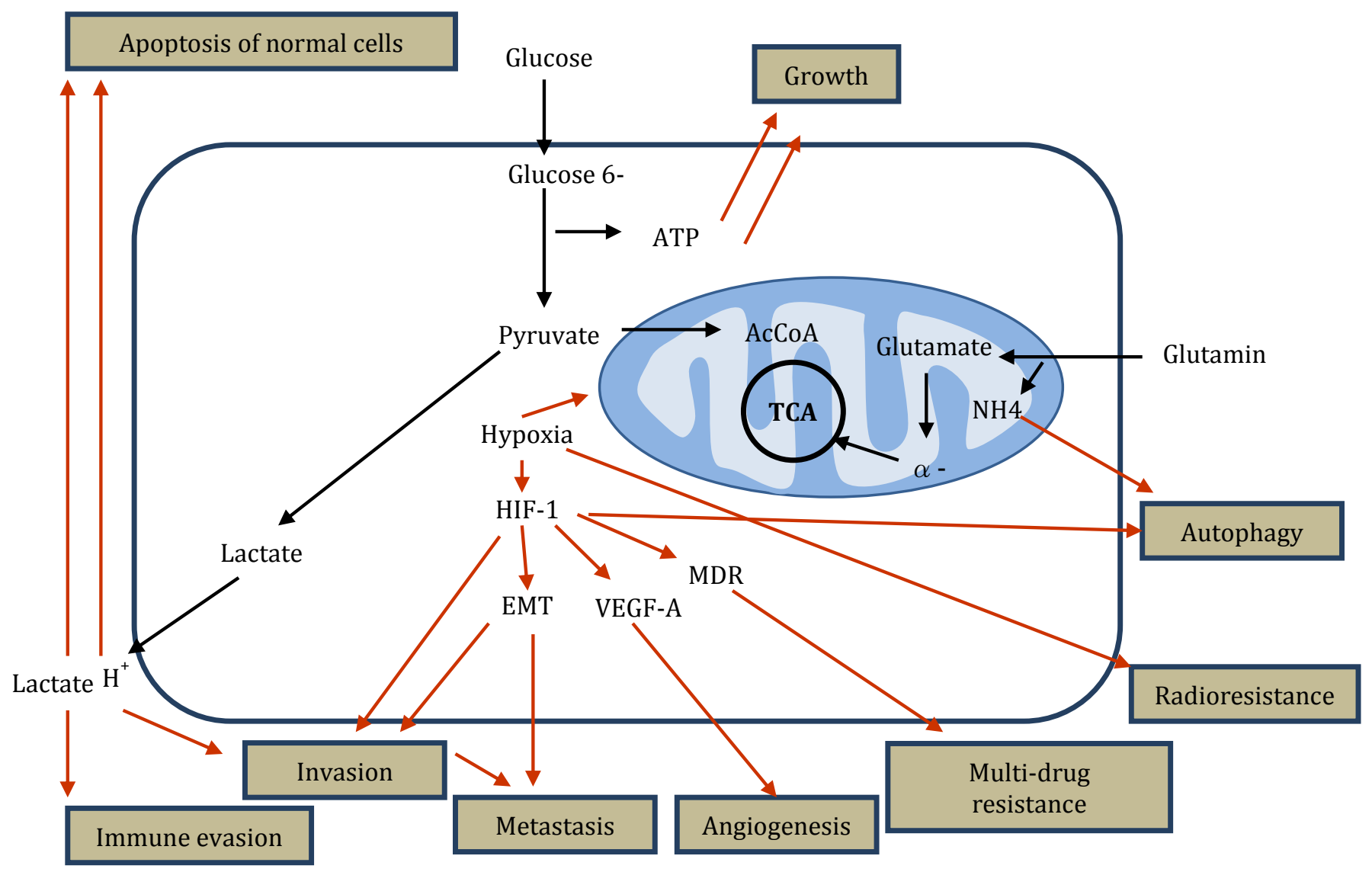

Figure 1. Possible targets of resveratrol and proposed roles of cancer metabolism in the expression of cancer hallmarks. The hallmarks concern the cancer cells themselves except apoptosis, which concerns neighboring normal cells. The glucose uptake rates of cancer cells are much higher than those of neighboring cells, leading to apoptosis of the latter. High glycolytic fluxes allow rapid processing of glucose into lactate, which, together with protons, induces apoptosis in normal cells, blocks immune cell function and guides tissue invasion by tumors. Hypoxia existing in tumors is not only responsible for tumor resistance, but also supports angiogenesis and metastasis through HIF-1 activity. Abbreviations: AcCoA; EMT, epithelial-mesenchymal 
transition; HIF-1, hypoxia inducible factor-1; $\alpha$-KG, $\alpha$-ketoglutarate; MDR, multi-drug resistance; TCA, tricarboxylic acid cycle;VEGF, vascular endothelial growth factor. Adapted from Ferreira et al. (2012) under permission from nature publishing group.

Anti-cancer mechanisms of resveratrol against hallmarks of Cancer - Inhibiting three key glycolytic enzymes: hexokinase, phosphofructokinase, and pyruvate kinase: Hexokinase (HK) catalyzes the essentially irreversible first step of the glycolytic pathway where glucose is phosphorylated to glucose-6-phosphate. Among four isoforms of HK, HK II is known to be overexpressed in most neoplastic cells where it contributes to the proliferation and survival of tumor cells through enhancing aerobic glycolysis [9]. There is no evidence suggesting that resveratrol directly inhibits the enhanced activity of HK II in tumor cells. However, growing evidence supports indirect HK II inhibition by resveratrol through the inhibitory effect of resveratrol on receptor tyrosine kinase (RTK)/phosphoinositide-3-kinase (PI3K)/AKT/mammalian target of rapamycin (mTOR) pathway in many types of cancer cells $[10,11]$. Resveratrol-induced HK II inhibition also seems to be mediated through the inhibition of hypoxia-inducible factor-1 $\alpha$ (HIF-1 $\alpha$ ), a transcription factor that reprograms cancer cell metabolism [12]. HIF-1 $\alpha$ overexpression is observed in a wide array of human cancers, which is known to be substantially responsible for the higher rate of glycolysis by the induction of glycolytic enzymes including HK II [13, 14]. Zhang et al. indicated that resveratrol treatment down-regulated the expression of HIF-1 $\alpha$ proteins in human nasopharyngeal carcinoma cells [15].

The enzyme 6-phosphofructo-1-kinase (PFK1) is another critical glycolytic enzyme which catalyzes the transfer of a phosphate from ATP to fructose-6-phosphate to produce fructose-1,6bisphosphate and adenosine diphosphate (ADP) [16]. The expression of PFK-L isoform was shown to correlate directly with aggressiveness and glycolytic efficiency, i.e., lactate produced per glucose consumed, in breast cancer cell lines [17]. Moreover, PFK1 inhibition can lead to cell death in human breast cancer cell lines, which suggests that PFK1 might play a critical role in cell fate decision in these cell lines [18]. Gomez et al. demonstrated that resveratrol decreased viability, glucose consumption and ATP content in human breast cancer cell line MCF-7, and these effects were correlated with PFK1 inhibition by resveratrol [16]. They also reported that resveratrol directly inhibited purified PFK1 mainly through the dissociation of the PFK1 from fully active tetramers into less active dimers.

Among the enzymes that undergo changes during tumor formation is pyruvate kinase (PK). PK catalyzes the dephosphorylation of phosphoenolpyruvate to pyruvate and yields one molecule of ATP [12]. In tumor cells, the glycolytic PK isoenzyme M2 (PKM2) determines whether glucose is converted to lactate for regeneration of energy (active tetrameric form, Warburg effect) or used for the synthesis of cell building blocks (nearly inactive dimeric form) [19]. Because of the lower glycolytic activity of PKM2 than the constitutively active isoform, PKM1, cancer cells are likely to accumulate several glycolytic intermediates for macromolecular biosynthesis, for example, ribose-5-phosphate using pentose phosphate pathway (PPP) [20]. mTOR up-regulates PKM2 via HIF-1 $\alpha$, while PKM2 conversely promotes transcriptional activity of HIF-1 $\alpha$, activating glycolysis at several levels and compensating the low glycolytic activity of PKM2 [21]. Resveratrol was shown to down-regulate PKM2 expression by inhibiting 
mTOR signaling. Down-regulation of PKM2 expression results in the suppression of cancer metabolism. Decreased glucose uptake and lactate production, and reduced anabolism were demonstrated in various cancer cell lines [20]. An over-expression of PKM2 abolished the effects of resveratrol, emphasizing the role of PKM2 down-regulation as a critical function of resveratrol.

Enhancing apoptotic cell death: Cancer cells are characterized by uncontrolled proliferation and defective apoptosis which contributes to chemotherapy resistance [22]. Changes of components of apoptotic machinery on mitochondrial membrane were suggested to be underlying mechanism of the defective apoptosis in cancer cells [9].

In addition to providing the precursor for glycolysis and biosynthesis of key metabolites, mitochondrial HK II may provide one link between inhibition of apoptosis and metabolic reprogramming in cancer cells through the interaction with the voltage-dependent anion channel (VDAC) on outer mitochondrial membrane (OMM) [22]. Partly due to the increased translocation of HK II to OMM by the constitutively activated Akt in cancer cells, the HK IIVDAC interaction was increased in cancer cells compared with normal cells. Furthermore, HK II was shown to bind more tightly to VDAC on OMM in tumor cells than in normal cells [23]. In addition to HK II-VDAC interaction, another critical regulation of the cellular apoptosis is the balance between pro- and anti-apoptotic proteins [24]. HK II may compete with the antiapoptotic protein $\mathrm{Bcl}-\mathrm{X}_{\mathrm{L}}$ for VDAC binding site. Thus, $\mathrm{HK}$ II binding to VDAC releases Bcl-X $\mathrm{X}_{\mathrm{L}}$ from VDAC and facilitates binding of $\mathrm{Bcl}-\mathrm{X}_{\mathrm{L}}$ to $\mathrm{Bax}$, which prevents Bax-Bax oligomerization or Bax-Bak interaction. However, HK II detachment from VDAC, for example, through phosphorylation of VDAC by glycogen synthase kinase $3 \beta$, promotes binding of $\mathrm{Bcl}-\mathrm{X}_{\mathrm{L}}$ to VDAC, leaving Bax free from Bcl- $\mathrm{X}_{\mathrm{L}}$. Free Bax interacts with Bak/Bax to form pore structures for the release of cytochrome $\mathrm{c}$, the commitment step of mitochondria-mediated apoptosis [25].

Resveratrol activity might reduce the translocation of HK II to OMM, thus preventing HK II-VDAC interaction and facilitating the detachment of HK II from VDAC via the inhibition of RTK/PI3K/AKT/mTOR pathway $[10,11]$. The detachment of HK II from the mitochondria might sensitize the mitochondria to the cisplatin-induced cell damage, leading to much enhanced apoptotic cell death [26]. Resveratrol was reported to induce apoptosis dominantly via the activation of Bak- but not Bax-mediated intrinsic pathway in human lung adenocarcinoma cells [27]. However, the growth-inhibitory and pro-apoptotic effects of piceatannol, a hydroxylated analog of resveratrol, were shown to be mediated through up-regulation of Bid, Bax. Bik, Bok, Fas: P21(WAF1), down-regulation of Bcl-xL; Bcl-2, clAP, activation of caspases (-3, -7,- 8, -9), loss of mitochondrial potential, and release of cytochrome $\mathrm{c}$ in a wide variety of tumor cells [28]. More recently, dimethylated analog of resveratrol, pterostilbene, was demonstrated to downregulate the anti-apoptotic proteins $\mathrm{Bcl}_{\mathrm{X}} \mathrm{L}$ and $\mathrm{Mcl}-1$, leading to the up-regulation of mitochondrial apoptosis pathway-related proteins including Bax, Bak, cytochrome c, and cleaved caspase 3 in osteosarcoma cells [29].

There is another mechanism of resveratrol enhancing apoptosis of cancer cells. Low $\mathrm{pH}$, caused by the accumulation of lactate due to up-regulated glycolysis in cancer cells, was shown to induce base rotation in DNA especially copper bound N7 guanine that can lead to exposure of the metal ion. Resveratrol seems to easily attack exposed copper, reducing it and thereby 
generating reactive oxygen species (ROS), leading to oxidative DNA damage [30]. These results suggest that resveratrol could be an effective treatment for tumors that are hypoxic and have low $\mathrm{pH}$ microenvironment.

Death receptor pathway, also known as the extrinsic pathway, is another apoptotic pathway through which the pro-apoptotic effect of resveratrol might be exhibited. Briefly, resveratrol can up-regulate the expression of death receptor ligands such as FasL and CD95L directly or indirectly through activating p53, leading to increase and relocalization of death receptors in lipid microdomains and forming the death-inducing signaling complex (DISC) [31]. The DISC complex recruits pro-caspase 8 through the adaptor protein Fas-associated death domain (FADD), activating downstream procaspases like caspase 3 to induce apoptosis [32].

Inhibiting proliferation of tumor cell: The anti-proliferative effect of resveratrol in tumor cells has been extensively described [33, 34]. In a wide variety of tumor cells, the growth-inhibitory effects of resveratrol were shown to be mediated through cell-cycle arrest. Most of the cell cycle is controlled by a family of protein kinase complexes which constitutes a cyclin dependent kinase (cdk) and the corresponding cyclin [31]. Wolter et al. showed the down-regulation of the cyclin D1/cdk 4 complex by resveratrol in colon cancer cells [35]. Resveratrol can also induce G1 phase arrest by decreasing the protein expression of cyclin D2, E, and cdk 2, 6 through inhibiting binding of transcription factors such as nuclear factor-kappa B (NF-kB) and activator protein-1 (AP-1). Resveratrol was shown to induce the translocation and expression of cdk inhibitors, p21 and p27, by up-regulation of transcription factors. Cdk inhibitors inhibit active cyclin/cdk complexes and block the G1/S phase transition [31]. Resveratrol can also decrease the expression of c-Myc, a cyclin E/cdk 2 activator, preventing the cell from going through G1/S phase transition [36]. There are several mechanism reported with regard to $S$ phase arrest by resveratrol. Resveratrol can disrupt the dephosphorylation of cdk 1, a key regulator of the cell cycle and the progression through the $\mathrm{S}$ phase [31]. Larrosa et al. reported that resveratrol induced S-phase arrest and up-regulation of cyclins A, E and B1 in human melanoma cells [37]. $S$ phase arrest by resveratrol was also induced by direct inhibition of DNA synthesis through, for example, inhibiting ribonucleotide synthase and DNA polymerases [38]. Liang et al. demonstrated that resveratrol induced G2 phase arrest through the inhibition of Cdk 7 and Cdc 2 kinases in colon carcinoma cells [39]. Thus, it seems to be clear that the resveratrol effects on the cell-cycle are highly variable [33].

Another mechanism of anti-proliferative effect of resveratrol was mediated through its role as a calorie restriction (CR) mimetic. CR is widely believed to extend the lifespan and protect against aging-related diseases including cancer [40]. Accumulating evidence showed that CR effect of resveratrol might be associated with increasing silent information regulator 2/sirtuin 1 (SIRT) activity [6]. SIRTs belong to the class III histone/protein deacetylases, which are implicated in CR, aging, and inflammation [41]. One key enzyme thought to be activated during CR is AMP-activated kinase (AMPK), a sensor of cellular energy levels [42]. Once activated, AMPK inhibited 4E-BP1 signaling and mRNA translation via mTOR, leading to inhibition of cellular proliferation. In addition, resveratrol-mediated AMPK activation was shown to be caused by inducing the expression of SIRT1 via elevation in the cellular NAD (+)/NADH in 
estrogen receptor-positive breast cancer cells. These findings suggest that targeting SIRT1/AMPK signaling by resveratrol may have potential therapeutic implications for cancer.

P53 is a key tumor suppressor protein that has multiple functions which help to prevent cancer development including regulation of cell growth as well as promoting apoptosis and dampening glycolysis [43]. Tumor cells often mutate or lose p53 to ensure replicative potential. Warburg effect is known to be directly caused by the inactivation of p53. P53 can inhibit the expression of glucose transporters (GLUT) 1 and 4, and phosphoglycerate mutase (PGM), the enzyme that converts 3-phosphoglycerate to 2-phosphoglycerate in glycolysis, while increasing the expression of TP53-induced glycolysis and apoptosis regulator (TIGAR) [22]. TIGAR is an isoform of PFK2 that inhibits overall PFK activity through lowering the levels of fructose-2, 6bisphosphate, an allosteric activator of PFK1, and hence inhibits glycolysis while channeling glucose to the PPP [44]. Again, PPP is one of the critical pathways for macromolecular biosynthesis which is needed to supply building blocks for cell replication. There are several lines of evidence demonstrating that resveratrol can up-regulate the expression of p53 in various cancer cell lines including breast, prostate, and ovarian cancers [45-47]. The underlying mechanism of p53 up-regulation by resveratrol is not clearly understood. Nevertheless, Zhang et al. proposed that resveratrol could activate p53 with the help of mitogen-activated protein kinase (MAPK), which phosphorylates and acetylates p53 [48].

\section{Angioprevention: anti- metastatic effect through inhibiting inflammation and angiogenesis:} It is now well established that metabolic syndrome is a risk factor for at least some types of cancers including breast, colon, and prostate cancers [5]. Among the involved pathological manifestations are inflammation and endothelial cell dysfunction. Obesity may shift the balance toward a pro-angiogenic and pro-inflammatory function, and become a reservoir for dysregulated inflammatory cells such as macrophages and T lymphocytes. These inflammatory cells are able to release cytokines for tumor promotion and angiogenesis [5]. The inflammatory mediators include cytokines such as interleukin (IL)-1, 6, 8, and tissue necrosis factor- $\alpha$ (TNF- $\alpha$ ), prostaglandins, and nitric oxide [49]. Cyclooxygenase (COX), which catalyzes the conversion of arachidonic acid to prostaglandins, was shown to be able to activate procarcinogens, thus, making transgenic mice with an increased expression of COX-2 highly susceptible to spontaneous skin tumor formation [50]. Surh et al. reported that COX-2 was implicated in increased invasiveness as well as metastatic potential of tumor cells [51]. Transcription of COX2 is regulated by several transcription factors including NF- $\kappa \mathrm{B}$ [31]. NF- $\kappa \mathrm{B}$, one of the most important transcription factors regulating inflammatory responses, cellular proliferation and growth as well as oncogenesis, is known to exist as a heterodimer and sterically blocked by IкB

[52]. The balance between NF- $\kappa \mathrm{B}$ and $\mathrm{I} \kappa \mathrm{B}$ was considered important to the tumor development [53].

Resveratrol was demonstrated to directly block NF- $\kappa \mathrm{B}$ activation in cell type non-specific manner [54]. In addition, accumulating evidence proposed several mechanisms underlying the $\mathrm{NF}-\kappa \mathrm{B}$ inhibition by resveratrol. Kundu et al. reported that resveratrol could prevent the activity of NF- $\kappa \mathrm{B}$ by inhibiting degradation of $\mathrm{I} \kappa \mathrm{B} \alpha$ in oxidative stress-stimulated mouse skin model [55]. MAPK phosphatase 5 (MAPK5) is known as a potent inhibitor of cellular inflammatory responses because MAPK5 can block the enzymatic activation of MAPK, which is one of the 
upstream kinases that regulate the activation of $\mathrm{NF}-\kappa \mathrm{B}$, with a high selectivity for $\mathrm{p} 38$. It was shown that resveratrol could up-regulate MAPK5 and inhibit p38 pathway in prostate cancer cell lines [56]. Moreover, resveratrol can inhibit NF- $\kappa \mathrm{B}$ both by blocking the upstream activator $\mathrm{PKC} \delta$ and by activating the inhibitor SIRT1 [57].

Angiogenesis is essential for tumor to survive and metastasize. It is usually required for tumor growth beyond a diameter 1-2 $\mathrm{mm}$ and stimulated by, most importantly, vascular endothelial growth factor (VEGF) [58]. Resveratrol was shown to inhibit tumor-induced neovascularization through inhibiting VEGF expression in various cancer cell lines $[59,60]$. The anti-angiogenic effect of resveratrol is also through inhibiting other mediators including basic fibroblast growth factor (bFGF) and matrix metalloproteinase-2, 9 (MMP-2, 9). MMPs are endopeptidases that cleave protein components of extracellular matix contributing to cell migration for angiogenesis as well as metastasis [61]. Resveratrol was shown to markedly inhibit MMP-9 activity and expression at both protein and mRNA levels in breast cancer cells [62]. In this study, resveratrol attenuated PI3K and phosphorylation of AKT and mTOR. In addition, resveratrol inhibited NF- $\kappa \mathrm{B}$ transcriptional activity and DNA binding of NF- $\kappa$ B on MMP-9 promoter. These results suggest that resveratrol may suppress breast cancer cell invasion through the inhibition of MMP-9 involved in PI3K/AKT and NF- $\kappa$ B pathways. Mousa et al. showed that resveratrol could inhibit bFGF-induced angiogenesis in the chick chorioallantoic membrane model [63].

Tosetti et al. have shown the capacity of dietary phytochemicals including resveratrol to inhibit inflammation and angiogenesis, termed angioprevention [5]. Along with down-regulation of NF- $\mathrm{BB}$ by resveratrol, a variety of inflammatory cytokines such as IL-1, 6, 8, and TNF- $\alpha$ were main targets of resveratrol [33]. Given that MAPK is a crucial component in both signaling pathways for inflammatory responses and endothelial cell proliferation, the inhibitory effect of resveratrol on MAPK suggests that resveratrol might play an important role in angioprevention to prevent tumor metastasis.

\section{CONCLUSIONS:}

Resveratrol is widely considered to exhibit beneficial health effects. Anti-cancer effects of resveratrol seem to be of particular interest at present, because resveratrol shares most of its targets with metabolic reprogramming of tumor cells. Metabolic reprogramming is a reemerging issue in recent cancer biology. Briefly, resveratrol down-regulates the increased glycolytic activity of tumor cells at multiple levels, facilitating apoptosis of tumor cells and inhibiting tumor cell proliferation. Moreover, resveratrol might prevent metastasis and tumor invasion through inhibiting inflammation and angiogenesis.

Of note, the anti-cancer effects of resveratrol are thought to have cancer selectivity with minimal toxicity to normal cells. Low $\mathrm{pH}$ condition around tumor cells due to increased lactate production from enhanced glycolysis may promote the pro-oxidant anti-cancer activity of resveratrol [30]. It should be pointed out that the IC50 of resveratrol for proliferation inhibition was significantly lower for cancer cells as compared to normal cells [64]. The cancer selectivity of resveratrol might help to circumvent its limit of low bioavailability. There are several clinical trials of resveratrol for testing its efficacy in prevention and treatment of cancer. We should wait for their results to find out whether the promising experimental and preclinical data will be 
proven in clinical setting.

Competing interests: The authors declare no conflict of interest.

Acknowledgements and Funding: This research was supported by Basic Science Research Program (No. 800-20120364), WCU (World Class University) program (R31-10056) and Priority Research Centers Program (No. 0431-20120003) through the National Research Foundation of Korea (NRF) funded by the Ministry of Education, Science and Technology. This work was also supported by grant from the SNUH Research Fund (No.0320120350 / No.3020110070 / No.0420100460).

\section{REFERENCES:}

1. Warburg O: On the origin of cancer cells. Science 1956, 123(3191):309-314.

2. Lopez-Lazaro M: The warburg effect: why and how do cancer cells activate glycolysis in the presence of oxygen? Anti-cancer agents in medicinal chemistry 2008, 8(3):305-312.

3. Vander Heiden MG, Cantley LC, Thompson CB: Understanding the Warburg effect: the metabolic requirements of cell proliferation. Science 2009, 324(5930):1029-1033.

4. Suh DH, Kim MK, Kim HS, Chung HH, Song YS: Unfolded protein response to autophagy as a promising druggable target for anticancer therapy. Annals of the New York Academy of Sciences 2012, 1271:20-32.

5. Tosetti F, Noonan DM, Albini A: Metabolic regulation and redox activity as mechanisms for angioprevention by dietary phytochemicals. International journal of cancer Journal international du cancer 2009, 125(9):1997-2003.

6. Holme AL, Pervaiz S: Resveratrol in cell fate decisions. Journal of bioenergetics and biomembranes 2007, 39(1):59-63.

7. Constant J: Alcohol, ischemic heart disease, and the French paradox. Coronary artery disease 1997, 8(10):645-649.

8. Jang M, Cai L, Udeani GO, Slowing KV, Thomas CF, Beecher CW, Fong HH, Farnsworth NR, Kinghorn AD, Mehta RG et al: Cancer chemopreventive activity of resveratrol, a natural product derived from grapes. Science 1997, 275(5297):218-220.

9. Suh DH, Kim MK, Kim HS, Chung HH, Song YS: Mitochondrial permeability transition pore as a selective target for anti-cancer therapy. Frontiers in oncology 2013, 3:41.

10. Castillo-Pichardo L, Dharmawardhane SF: Grape polyphenols inhibit Akt/mammalian target of rapamycin signaling and potentiate the effects of gefitinib in breast cancer. Nutrition and cancer 2012, 64(7):1058-1069.

11. Jiang H, Shang X, Wu H, Gautam SC, Al-Holou S, Li C, Kuo J, Zhang L, Chopp M: Resveratrol downregulates PI3K/Akt/mTOR signaling pathways in human U251 glioma cells. Journal of experimental therapeutics \& oncology 2009, 8(1):25-33.

12. Sun Q, Chen X, Ma J, Peng H, Wang F, Zha X, Wang Y, Jing Y, Yang H, Chen R et al: Mammalian target of rapamycin up-regulation of pyruvate kinase isoenzyme type M2 is 
critical for aerobic glycolysis and tumor growth. Proceedings of the National Academy of Sciences of the United States of America 2011, 108(10):4129-4134.

13. Wenner CE: Targeting mitochondria as a therapeutic target in cancer. Journal of cellular physiology 2012, 227(2):450-456.

14. Semenza GL: Targeting HIF-1 for cancer therapy. Nature Reviews Cancer 2003, 3(10):721-732.

15. Zhang M, Zhou X, Zhou K: Resveratrol inhibits human nasopharyngeal carcinoma cell growth via blocking pAkt/p70S6K signaling pathways. International journal of molecular medicine 2013, 31(3):621-627.

16. Gomez LS, Zancan P, Marcondes MC, Ramos-Santos L, Meyer-Fernandes JR, Sola-Penna M, Da Silva D: Resveratrol decreases breast cancer cell viability and glucose metabolism by inhibiting 6-phosphofructo-1-kinase. Biochimie 2013.

17. Zancan P, Sola-Penna M, Furtado CM, Da Silva D: Differential expression of phosphofructokinase-1 isoforms correlates with the glycolytic efficiency of breast cancer cells. Molecular genetics and metabolism 2010, 100(4):372-378.

18. Furtado CM, Marcondes MC, Sola-Penna M, de Souza ML, Zancan P: Clotrimazole preferentially inhibits human breast cancer cell proliferation, viability and glycolysis. PloS one 2012, 7(2):e30462.

19. Mazurek S: Pyruvate kinase type M2: a key regulator of the metabolic budget system in tumor cells. The international journal of biochemistry \& cell biology 2011, 43(7):969-980.

20. Iqbal MA, Bamezai RN: Resveratrol inhibits cancer cell metabolism by down regulating pyruvate kinase M2 via inhibition of mammalian target of rapamycin. PloS one 2012, 7(5):e36764.

21. Munoz-Pinedo C, El Mjiyad N, Ricci JE: Cancer metabolism: current perspectives and future directions. Cell death \& disease 2012, 3:e248.

22. Kroemer G, Pouyssegur J: Tumor cell metabolism: cancer's Achilles' heel. Cancer cell 2008, 13(6):472-482.

23. Pedersen PL: Warburg, me and Hexokinase 2: Multiple discoveries of key molecular events underlying one of cancers' most common phenotypes, the "Warburg Effect", i.e., elevated glycolysis in the presence of oxygen. Journal of bioenergetics and biomembranes 2007, 39(3):211-222.

24. Gogvadze V, Orrenius S, Zhivotovsky B: Mitochondria as targets for chemotherapy. Apoptosis : an international journal on programmed cell death 2009, 14(4):624-640.

25. Pastorino JG, Hoek JB: Regulation of hexokinase binding to VDAC. Journal of bioenergetics and biomembranes 2008, 40(3):171-182.

26. Shulga N, Wilson-Smith R, Pastorino JG: Hexokinase II detachment from the mitochondria potentiates cisplatin induced cytotoxicity through a caspase-2 dependent mechanism. Cell cycle 2009, 8(20):3355-3364.

27. Zhang $\mathrm{W}$, Wang $\mathrm{X}$, Chen $\mathrm{T}$ : Resveratrol induces apoptosis via a Bak-mediated intrinsic pathway in human lung adenocarcinoma cells. Cellular signalling 2012, 24(5):1037-1046.

28. Piotrowska H, Kucinska M, Murias M: Biological activity of piceatannol: leaving the shadow of resveratrol. Mutation research 2012, 750(1):60-82. 
29. Liu Y, Wang L, Wu Y, Lv C, Li X, Cao X, Yang M, Feng D, Luo Z: Pterostilbene exerts antitumor activity against human osteosarcoma cells by inhibiting the JAK2/STAT3 signaling pathway. Toxicology 2013, 304:120-131.

30. Muqbil I, Beck FW, Bao B, Sarkar FH, Mohammad RM, Hadi SM, Azmi AS: Old wine in a new bottle: the Warburg effect and anticancer mechanisms of resveratrol. Current pharmaceutical design 2012, 18(12):1645-1654.

31. Kraft TE, Parisotto D, Schempp C, Efferth T: Fighting cancer with red wine? Molecular mechanisms of resveratrol. Critical reviews in food science and nutrition 2009, 49(9):782799.

32. Delmas D, Rebe C, Lacour S, Filomenko R, Athias A, Gambert P, Cherkaoui-Malki M, Jannin B, Dubrez-Daloz L, Latruffe N et al: Resveratrol-induced apoptosis is associated with Fas redistribution in the rafts and the formation of a death-inducing signaling complex in colon cancer cells. The Journal of biological chemistry 2003, 278(42):41482-41490.

33. Aggarwal BB, Bhardwaj A, Aggarwal RS, Seeram NP, Shishodia S, Takada Y: Role of resveratrol in prevention and therapy of cancer: preclinical and clinical studies. Anticancer research 2004, 24(5A):2783-2840.

34. Yu L, Sun ZJ, Wu SL, Pan CE: Effect of resveratrol on cell cycle proteins in murine transplantable liver cancer. World journal of gastroenterology : WJG 2003, 9(10):23412343.

35. Wolter F, Akoglu B, Clausnitzer A, Stein J: Downregulation of the cyclin D1/Cdk4 complex occurs during resveratrol-induced cell cycle arrest in colon cancer cell lines. The Journal of nutrition 2001, 131(8):2197-2203.

36. Zhang P, Li H, Wu ML, Chen XY, Kong QY, Wang XW, Sun Y, Wen S, Liu J: c-Myc downregulation: a critical molecular event in resveratrol-induced cell cycle arrest and apoptosis of human medulloblastoma cells. Journal of neuro-oncology 2006, 80(2):123131.

37. Larrosa M, Tomas-Barberan FA, Espin JC: Grape polyphenol resveratrol and the related molecule 4-hydroxystilbene induce growth inhibition, apoptosis, S-phase arrest, and upregulation of cyclins A, E, and B1 in human SK-Mel-28 melanoma cells. Journal of agricultural and food chemistry 2003, 51(16):4576-4584.

38. Stivala LA, Savio M, Carafoli F, Perucca P, Bianchi L, Maga G, Forti L, Pagnoni UM, Albini A, Prosperi E et al: Specific structural determinants are responsible for the antioxidant activity and the cell cycle effects of resveratrol. The Journal of biological chemistry 2001, 276(25):22586-22594.

39. Liang YC, Tsai SH, Chen L, Lin-Shiau SY, Lin JK: Resveratrol-induced G2 arrest through the inhibition of CDK7 and p34CDC2 kinases in colon carcinoma HT29 cells. Biochemical pharmacology 2003, 65(7):1053-1060.

40. Chung JH, Manganiello V, Dyck JR: Resveratrol as a calorie restriction mimetic: therapeutic implications. Trends in cell biology 2012, 22(10):546-554.

41. Cosgrove MS, Bever K, Avalos JL, Muhammad S, Zhang X, Wolberger C: The structural basis of sirtuin substrate affinity. Biochemistry 2006, 45(24):7511-7521. 
42. Lin JN, Lin VC, Rau KM, Shieh PC, Kuo DH, Shieh JC, Chen WJ, Tsai SC, Way TD: Resveratrol modulates tumor cell proliferation and protein translation via SIRT1-dependent AMPK activation. Journal of agricultural and food chemistry 2010, 58(3):1584-1592.

43. Vousden KH, Ryan KM: p53 and metabolism. Nature reviews Cancer 2009, 9(10):691700.

44. Bensaad K, Tsuruta A, Selak MA, Vidal MN, Nakano K, Bartrons R, Gottlieb E, Vousden KH: TIGAR, a p53-inducible regulator of glycolysis and apoptosis. Cell 2006, 126(1):107120.

45. Narayanan BA, Narayanan NK, Re GG, Nixon DW: Differential expression of genes induced by resveratrol in LNCaP cells: P53-mediated molecular targets. International journal of cancer Journal international du cancer 2003, 104(2):204-212.

46. Piotrowska H, Myszkowski K, Ziolkowska A, Kulcenty K, Wierzchowski M, Kaczmarek M, Murias M, Kwiatkowska-Borowczyk E, Jodynis-Liebert J: Resveratrol analogue 3,4,4',5-tetramethoxystilbene inhibits growth, arrests cell cycle and induces apoptosis in ovarian SKOV-3 and A-2780 cancer cells. Toxicology and applied pharmacology 2012, 263(1):53-60.

47. Maccario C, Savio M, Ferraro D, Bianchi L, Pizzala R, Pretali L, Forti L, Stivala LA: The resveratrol analog 4,4'-dihydroxy-trans-stilbene suppresses transformation in normal mouse fibroblasts and inhibits proliferation and invasion of human breast cancer cells. Carcinogenesis 2012, 33(11):2172-2180.

48. Zhang S, Cao HJ, Davis FB, Tang HY, Davis PJ, Lin HY: Oestrogen inhibits resveratrolinduced post-translational modification of p53 and apoptosis in breast cancer cells. British journal of cancer 2004, 91(1):178-185.

49. Coussens LM, Werb Z: Inflammation and cancer. Nature 2002, 420(6917):860-867.

50. Muller-Decker K, Neufang G, Berger I, Neumann M, Marks F, Furstenberger G: Transgenic cyclooxygenase-2 overexpression sensitizes mouse skin for carcinogenesis. Proceedings of the National Academy of Sciences of the United States of America 2002, 99(19):12483-12488.

51. Surh YJ, Chun KS, Cha HH, Han SS, Keum YS, Park KK, Lee SS: Molecular mechanisms underlying chemopreventive activities of anti-inflammatory phytochemicals: downregulation of COX-2 and iNOS through suppression of NF-kappa B activation. Mutation research 2001, 480-481:243-268.

52. Han SS, Keum YS, Seo HJ, Chun KS, Lee SS, Surh YJ: Capsaicin suppresses phorbol ester-induced activation of NF-kappaB/Rel and AP-1 transcription factors in mouse epidermis. Cancer letters 2001, 164(2):119-126.

53. Aggarwal BB: Signalling pathways of the TNF superfamily: a double-edged sword. Nature reviews Immunology 2003, 3(9):745-756.

54. Manna SK, Mukhopadhyay A, Aggarwal BB: Resveratrol suppresses TNF-induced activation of nuclear transcription factors NF-kappa B, activator protein-1, and apoptosis: potential role of reactive oxygen intermediates and lipid peroxidation. Journal of immunology 2000, 164(12):6509-6519. 
55. Kundu JK, Shin YK, Kim SH, Surh YJ: Resveratrol inhibits phorbol ester-induced expression of COX-2 and activation of NF-kappaB in mouse skin by blocking IkappaB kinase activity. Carcinogenesis 2006, 27(7):1465-1474.

56. Nonn L, Duong D, Peehl DM: Chemopreventive anti-inflammatory activities of curcumin and other phytochemicals mediated by MAP kinase phosphatase-5 in prostate cells. Carcinogenesis 2007, 28(6):1188-1196.

57. Yeung F, Hoberg JE, Ramsey CS, Keller MD, Jones DR, Frye RA, Mayo MW: Modulation of NF-kappaB-dependent transcription and cell survival by the SIRT1 deacetylase. The EMBO journal 2004, 23(12):2369-2380.

58. Tseng SH, Lin SM, Chen JC, Su YH, Huang HY, Chen CK, Lin PY, Chen Y: Resveratrol suppresses the angiogenesis and tumor growth of gliomas in rats. Clinical cancer research : an official journal of the American Association for Cancer Research 2004, 10(6):21902202.

59. Yang R, Zhang H, Zhu L: Inhibitory effect of resveratrol on the expression of the VEGF gene and proliferation in renal cancer cells. Molecular medicine reports 2011, 4(5):981983.

60. Yu HB, Zhang HF, Zhang X, Li DY, Xue HZ, Pan CE, Zhao SH: Resveratrol inhibits VEGF expression of human hepatocellular carcinoma cells through a NF-kappa Bmediated mechanism. Hepato-gastroenterology 2010, 57(102-103):1241-1246.

61. Bogaczewicz J, Jasielski P, Mosiewicz A, Trojanowski T, Suchozebrska-Jesionek D, Stryjecka-Zimmer M: [The role of matrix metalloproteinases and tissue inhibitors of metalloproteinases in invasion of tumours of neuroepithelial tissue]. Neurologia $i$ neurochirurgia polska 2006, 40(5):404-412.

62. Ko HS, Lee HJ, Kim SH, Lee EO: Piceatannol suppresses breast cancer cell invasion through the inhibition of MMP-9: involvement of PI3K/AKT and NF-kappaB pathways. Journal of agricultural and food chemistry 2012, 60(16):4083-4089.

63. Mousa SS, Mousa SS, Mousa SA: Effect of resveratrol on angiogenesis and platelet/fibrinaccelerated tumor growth in the chick chorioallantoic membrane model. Nutrition and cancer 2005, 52(1):59-65.

64. Gautam SC, Xu YX, Dumaguin M, Janakiraman N, Chapman RA: Resveratrol selectively inhibits leukemia cells: a prospective agent for ex vivo bone marrow purging. Bone marrow transplantation 2000, 25(6):639-645. 